\title{
La femme dans la littérature et la culture russes du début du $\mathrm{XX}^{\mathrm{e}}$ siècle
}

Sujet exotique et objet d'expérience exotique

\section{Sergueï Savinkov}

Traducteur : Corinne Fournier Kiss

\section{(2) OpenEdition}

1 Journals

Édition électronique

URL : http://journals.openedition.org/edl/422

DOI : 10.4000/edl.422

ISSN : 2296-5084

Éditeur

Université de Lausanne

\section{Édition imprimée}

Date de publication : 15 septembre 2009

Pagination : 157-172

ISBN : 978-2-940331-20-8

ISSN : 0014-2026

Référence électronique

Sergueï Savinkov, "La femme dans la littérature et la culture russes du début du XX siècle ", Études de lettres [En ligne], 2-3|2009, mis en ligne le 15 septembre 2012, consulté le 21 décembre 2020. URL : http://journals.openedition.org/edl/422 ; DOI : https://doi.org/10.4000/edl.422 


\section{LA FEMME DANS LA LITTÉRATURE ET LA CULTURE RUSSES DU DÉBUT DU XX $X^{\mathrm{e}}$ SIÈCLE: SUJET EXOTIQUE ET OBJET D'EXPÉRIENCE EXOTIQUE}

La littérature russe de la période fin de siècle explore avec passion la femme, à la fois métaphore et métonymie des rapports entre l'être humain et l'univers, l'individu et la société, le corps et l'esprit. L'article examine les figures antithétiques de Lilith et d'Eve ainsi que celle, intermédiaire, de Salomé, tantôt femme vampire, tantôt garante "dionysiaque» de la fusion avec le monde. L'opposition entre la proximité familière et l'éloignement exotique organise ces symboliques interconnectées.

\section{Lilith et Eve, la femme-rêve et la femme-vie}

L'idée principale de l'article de N. Wrangel «Le rêve préféré des peintres russes contemporains. Les Madones» ${ }^{1}$ peut se résumer ainsi : dans la littérature et dans les arts plastiques du début du $\mathrm{XX}^{\mathrm{e}}$ siècle, le rêve de la femme du Paradis se cristallise désormais non plus sur l'image de la Vierge Marie, «icône au visage d'Eve, femme terrestre», mais sur l'image de l'autre, celle qu'une légende présente comme la première épouse d'Adam, et une seconde légende comme un hideux «démon de la nuit».

Comment expliquer cette fascination de l'époque pour le personnage de Lilith? Lilith fait son entrée dans la littérature du début du XXe siècle en tant qu'incarnation du principe inverse de celui représenté par Eve. Lilith, en effet, s'oppose à Eve de la même façon que le rêve à la réalité.

I. N. N. Vrangel', «Ljubovnaja mečta sovremennyh russkih hudožnikov». 
Montrer sa fascination pour Lilith, c'est donc exprimer de manière indirecte sa répugnance envers l'Eve principe de vie ou, pour reprendre les termes de Wrangel, envers tout ce qui «est toujours avec nous», ce qui nous colle à la peau comme le quotidien, la routine, la mesure, tout ce qui est sain. On observe souvent dans le rêve de Lilith des traits décadents :

Dans la peinture fait son apparition, de manière encore plus saisissante que dans la littérature, un nouveau type de femme désirée, femme chétive, rêveuse et triste, issue d'un mélange de sentimentalisme remontant à l'époque de la "pauvre Lise», et des rêves dépravés de la modernité. Cette étrange combinaison de péché et de pureté naïve engendre une rêverie intense et oppressante ${ }^{2}$.

Toutefois, il ne s'agit pas seulement, ici, de rêve et de réalité, mais de rêve sous le signe de Lilith et de réalité sous le signe d'Eve, donc pas uniquement de l'opposition traditionnelle entre les deux femmes. Lilith est une vierge lunaire, non par nature, mais par la force des circonstances: lunaire, elle l'est devenue, alors qu'elle aurait parfaitement pu être terrestre. Et ce conditionnel, cet "aurait pu», apporte aux émotions des individus du tournant du siècle un élément nouveau, ignoré de la grammaire romantique.

L'homme fin de siècle vit avec le sentiment d'une constante substitution. Tel lieu assigné à tel être se voit occupé par un autre. "Pourquoi n'es-tu pas avec moi, ma Lilith de minuit ?» ${ }^{3}$ demande Adam, le héros lyrique de Fiodor Sologoub. Et chez Marina Tsvetaeva, c'est Lilith ellemême qui pose la question à l'Adam moderne: pourquoi m'as-tu changée pour Eve? La «tentative de jalousie» tsvetaevienne est une série de questions sans réponse posées par une Lilith humiliée, offensée et désemparée parce qu’on lui en a préféré une autre:

2. «V živopisi ešče jarče, čem v literature, vyrastaet novyj tip želannoj ženščiny, bol'noj, mečtatel'noj i grustnoj, smes' sentimentalizma vremen "bednoj Lizy” s poročnymi mečtami sovremennosti. I strannoe sočetanie greha i naivnoj čistoty proizvodit ostroe i ščemjaščee mečtanie. » Ibid., p. 38. Bednaja Liza (Pauvre Lise, 1789): récit de Nikolaï Karamzine, chef de file des pré-romantiques russes.

3. F. Sologub, Sobr. soč., t. 7, p. 492. 
«Comment est la vie avec l'autre, l'étrangère, l'autochtone... ? [...] Comment est la vie avec un simulacre...? [...] Comment vous portezvous avec la cent-millième - vous qui avez connu Lilith?» ${ }^{4}$

Lilith apparaît ici, comme la personnification, non de ce qu'on laisse échapper, mais de ce que l'on refuse et ignore, inconscient de ce que l'on perd en portant son choix sur l'autre, sur l'Eve ordinaire et terrestre. Car l'on perd vraiment quelque chose, si l'on en croit Marina Tsvetaeva: «le sixième sens", donc, du même coup, les hauteurs du Sinaï et Carrare.

\section{La première femme d'Adam}

La primauté de Lilith ne pouvait que s'exprimer dans son destin culturel et littéraire au tournant du siècle. Selon la légende, le statut de Lilith en tant que première femme ne dépend pas que de la chronologie, mais aussi du fait que, contrairement à Eve qui fut créée par Dieu à partir d'une côte d'Adam, elle fut pétrie de la même argile que son époux. De là sa prétention, dans ses relations avec le sexe opposé, sinon à la supériorité, du moins à une égalité sans condition et, par extension, à la liberté individuelle et à la liberté d'expression. Dans la littérature fin de siècle, ces exigences de liberté (qui ont par ailleurs nourri les mouvements féministes) sont patentes: en témoigne la quantité impressionnante de poésie spécifiquement féminine qui voit le jour à cette époque; en témoignent encore les nets penchants à la féminité et à l'amour saphique qui s'y expriment. "Je vous aime» - c'est par ces mots que Marina Tsvetaeva commence le premier poème du cycle «L'Amie», et elle le termine ainsi :

Pour ce frisson, pour ce: serait-ce un songe?, pour l'ironie délicieuse qui veut que Vous n'êtes pas «il» 5 .

Toute la poésie amoureuse de Sophie Parnok parle avec la même «délicieuse ironie» de l'objet de son amour, qui désormais n'est point «lui».

4. «Kak živetsja vam s čužoju, zdešneju...? [...] Kak živetsja vam s podobiem...? [...] Kak živetsja vam s tovarom rynočnym...? [...] Kak živetsja vam s sto-tysjačnoj Vam, poznavšemu Lilit?», M. Cvetaeva, Sob. soč v 7 tt., t. 2, p. 242-243.

5. Ibid., t. 1, p. 216. 
Dans ces «délices", toutefois, on sent une pointe de souffrance. La possibilité même d'une telle substitution - "Vous n'êtes pas “il”" - n'a pu se concrétiser que parce que ce «il» a préféré à Lilith l'unique, l'incomparable et l'indéfinissable, avec laquelle Marina Tsvetaeva s'identifie partiellement - à la "cent-millième» Eve. Dans une lettre à Boris Pasternak, elle avoue:

Boris, te souviens-tu de Lilith ?... Ton mal de moi, c'est le désir d'Adam pour Lilith, la première et l'indéfinissable (De là ma haine pour Eve!) ${ }^{6}$.

Contrairement à la tradition, Lilith est souvent perçue et représentée dans la littérature du début du $\mathrm{XX}^{\mathrm{e}}$ siècle comme une figure de souffrance passive. Cette image a été hautement favorisée par l'un des promoteurs de la décadence dans la littérature et la vie ("Colomb lunaire, qui par la force de l'esprit fit naître un rêve: Lilith $»^{7}$ ): Fiodor Sologoub.

Dans l'œuvre de Sologoub, la passivité de Lilith est conditionnée par le rôle que lui a dévolu le destin : être la première femme d'Adam. Ses principales qualités ne sont pas l'orgueil et le caprice de la Lilith mythique - celle qui quitte Adam en riant et se refuse à jouer auprès de lui le rôle de la «seconde»-, mais l'humilité et la résignation. Tout se passe comme si cette "autre» Lilith-la première, ne venait à Adam que pour mieux s'en aller et mieux laisser la place à sa femme légitime, Eve. Sa première place est marquée au coin de la précarité et de l'éphémère:

Quand le jour d'azur a pâli, Quand l'aube s'est inclinée vers les mers, Ma Lilith a passé telle une ombre, elle a passé, elle est partie - elle s'est cachée à jamais ${ }^{8}$.

Dans le rêve sologoubien de Lilith, on perçoit la nostalgie tsvetaevienne de l'unité perdue de l'androgyne, alliée à l'indignation et la perplexité devant la substitution accomplie: pourquoi ce qui devait être proche estil devenu lointain, et pourquoi ce qui devait être lointain est-il devenu proche: «Pourquoi donc n'es-tu pas avec moi, ma Lilith de minuit?»

6. Ibid., t. 6, p. 244.

7. C'est ainsi qu'une des nombreuses admiratrices et correspondantes de Sologoub le qualifia. T. V. Misnikevič, «Fedor Sologub, ego poklonnicy i korrespondentki», p. 374 .

8. F. Sologub, Sobr. soč., t. 7, p. 140. 


\section{Lilith ou le premier amour}

Dans la grammaire des émotions fin de siècle, l'imperfectif est préféré au perfectif, et le premier amour sans suite au second. Le héros du poème d'Igor Severianine, "La rosée de l'heure orange», parle de son premier amour comme d'un rêve voué à demeurer tel. Son premier amour, qu'il appelle «ma Lilith», lui a donné un avant-goût du paradis, mais un paradis dont les portes lui étaient, d'emblée, fermées:

«Mon cher, tu as tort: tu ne peux m'aimer ainsi... Tu n'oses pas... tu ne dois pas... C'est en vain que tu te berces et te tourmentes de rêves: ces songes, hélas, ne seront jamais que des songes, - Je ne peux... je ne dois point t'aimer... comme une épouse...» - Puis elle vient à moi, rafraîchit ma fièvre de ses lèvres en me baisant tendrement le front, comme une sœur, et je sanglote: l'éden m’est à jamais fermé?

La Lilith rêvée est sincère et bienveillante. Elle ne trompe ni ne séduit. Elle ne cache pas son inaccessibilité. La Lilith chimère se comporte, elle, de manière bien plus raffinée: c'est par son apparente accessibilité qu'elle charme et attire.

Contrairement à la Lilith de Severianine, celle de Nabokov ${ }^{10}$ promet à l'homme, seulement après la mort, d'exaucer son désir le plus secret, le premier appel du sexe, et le conduit à la félicité; mais ensuite, elle le précipite dans l'abîme en rendant impossible l'aboutissement de ce qui était en cours de réalisation. Ainsi l'homme devant lequel se ferment, au dernier instant, les portes du paradis, comprend-il pleinement ce qu'est l'enfer:

Le visage renversé était charmant et heureux, et, d'un énergique coup de reins, je pénétrai l'inoubliable... Lors, plein de force, à mi-chemin du bonheur, je me retrouvai sans rien... Laisse-moi entrer... La porte, obstinément, se taisait... Et, devant tout le monde, je versai douloureusement ma semence et compris soudain que j'étais en enfer ${ }^{11}$.

9. «"Milyj, ty ne prav: Tak ty ljubit' menja ne možeš’... Ne smeeš”... ty ne dolžen... ty / Naprasno greziš’ I trevožišs / Sebja mečtami : te mečty, / Uvy ostanutsja mečtami, - Ja ne mogu... ja ne dolžna Tebja ljubit'... nu, kak žena... ” - / I podojdja ko mne, ustami / Žar ohlaždaet moj ona, / Menja v čelo celuja nežno, / Po-sestrinski, I ja navzryd / Rydaju: raj navek zakryt», I. Severjanin, Stihotvorenija i poèmy, p. 323.

Io. Il s'agit de l'hérö̈ne du poème «Lilith» dans Poems and Problems de Vladimir Nabokov.

II. V. Nabokov, Stihotvorenija i poèmy, p. 323. 
Beaucoup plus tard, dans le roman Lolita, Nabokov reproduit la situation thématique de son poème, mais il place les accents ailleurs. Son héros est obsédé par l'idée d'accomplir ce qui est impossible. Lolita apparaît dans sa vie comme la réincarnation de l'Annabelle de son enfance, qui (sur le modèle des premières femmes de Sologoub et de Poe), ayant quitté la vie très tôt, sans avoir le temps de lui être ni une femme ni une épouse, lui laisse, cependant, une sensation de béatitude, incompréhensible et inaccessible. Le souvenir inoubliable de cette union presque androgyne fait naître chez le protagoniste de Nabokov l'idée fixe que cette félicité pourrait à nouveau être éprouvée, non pas avec une femme adulte, mais uniquement avec une nymphette à la frontière entre le monde de l'enfance et celui de la maturité. "Humbert était parfaitement capable d'avoir une relation avec Eve, mais il ne rêvait que de Lilith" ${ }^{12}$.

Ce que le héros de Nabokov craint le plus (et qu'en même temps il désire comme une délivrance), se réalise finalement: Lolita dépasse le stade de la nymphette pour devenir une Eve, et Humbert la retrouve précisément au moment où la grossesse détruit son image de nymphette: ventre proéminent, taches de rousseur pâlissantes et joues creuses. Mais c'est aussi au moment où il la voit, portant l'enfant d'un autre dans son sein, qu'il découvre en elle une autre beauté à laquelle il n'avait jamais prêté attention: non pas celle de Vénus Ourania ni de Vénus Pandemos, mais une beauté pâle et souillée, et en même temps pleine de douceur:

C'est maintenant seulement - à cette heure désespérée et tardive de ma vie - que je vois combien elle ressemble - et a toujours ressemblé à la blonde Vénus de Botticelli ${ }^{13}$.

\section{Eve ou Lilith}

"Eve ou Lilith", tel est le titre d'un poème de Nikolaï Goumilev dans lequel la conjonction disjonctive désigne un autre point important de la grammaire des émotions de l'époque: ce «ou» trace moins un trait de séparation qu'il ne rend floue la frontière entre les deux termes. Aussi l'héroïne ne semble-t-elle pas en mesure de dire avec précision qui elle

I2. Ibid., p. 30.

I3. Ibid., p. 311. 
est: "Tu ne te connais pas encore toi-même. Es-tu Eve ou Lilith?». Et bien que la jeune fille ait entendu maints récits sur sa grand-mère Eve, gardienne du foyer, sans connaître aucune légende sur Lilith, elle n'en éprouve pas moins pour cette dernière un sentiment de proximité et de parenté. Elle ne se languit point «des enfants, du troupeau de moutons et des pommes de terre", elle aspire "à une âme ailée et à la liberté de l'éden", ou encore à ce charme exotique, qu'on ne trouve pas chez Eve, mais qui appartient pleinement à Lilith («Lilith possède une couronne d'inaccessibles constellations, / Dans ses pays fleurissent des soleils de diamant " ${ }^{14}$ ). La reconnaissance définitive de soi comme Lilith (ou de Lilith en soi) ne se produira chez l'héroïne de Goumilev que lorsque fera irruption dans sa vie celui dont le cœur n'est pas une fleur, mais un météore:

Oh, quand viendra-t-il, Celui qui mènera

Ton cœur timide et avide dans la grotte enchantée?...

$S$ 'il le faut, il conquerra pour toi un royaume,

S'il le faut, il deviendra bandit de grand chemin. ${ }^{15}$

Le héros, à "l'âme constellée de pensées et de souffrances», viendra et sera en mesure de protéger Lilith des menées d'Eve:

Toujours et partout il la défendra contre Eve,

La défendra contre elle-même ${ }^{16}$.

La duplicité de l'héroïne de Goumilev est d'une autre nature que celle des jeunes filles hypocrites de Lermontov. Elle ne s'enracine ni dans la tromperie ni dans l'illusion («Belle comme un ange céleste, / Perfide et méchante comme un démon»), mais est à comprendre comme l'expression pure des deux principes en lutte dans la nature féminine depuis les origines. Même quand Eve remporte la victoire, rien ne garantit que Lilith ne puisse se réveiller et s'affirmer. Ce thème est développé chez

I4. "U Lillit nedostupnyh sozvezdij venec. V ee stranah almaznye solnca cvetut", N. Gumilev, Sočinenija v 3 tt., t. 1, p. 449.

I5. "O, kogda on pridet, / Tot, kto robkoe žadnoe serdce tvoe / Bez dorog uneset v začarovannyj grot?... / Esli nado, on carstvo tebe pokorit, / Esli nado, pojdet s vorovskoju sumoj».

I6. Ibid., p. 450. «No vsegda i povsjudu - ot Evy Lilit / On tebja sohranit ot tebja že samoj.» 
Goumilev dans le poème "Le rêve d'Adam». Anna Akhmatova en a donné le commentaire suivant:

Dans le «Rêve d'Adam» ressurgit ce "problème de la femme» (Eve et Lilith - la sainte et la pécheresse). Et l'effroi : «Une étrangère, une étrangère» ${ }^{17}$.

Dans le «Rêve d'Adam", le premier homme, endormi près des portes de la connaissance, a de son avenir une vision de cauchemar. L'horreur ne porte pas sur les peines et les privations qui l'attendent, mais sur le doute lancinant qui le taraude à l'égard de la femme qui se tient à ses côtés: la connaît-il vraiment? Est-elle vraiment ce qu'elle paraît, et non une autre? Est-elle vraiment sienne, et non étrangère? C'est dans l'absence de telles précisions que réside, pour Goumiliev, toute l'angoisse de l'amour ${ }^{18}$.

Et la douce Eve, ce jouet des dieux, naguère enfant, naguère aurore,

Est pour lui maintenant une jeune tigresse,

Dans le scintillement funeste de ses perles,

Présage de tempête, de sang et de passion

Et de mauvaises joies, et de sombres malheurs ${ }^{19}$.

Le châtiment d'Adam est de devoir mener une lutte épuisante et stérile dans le but de s'approprier ce qui, de toute façon, ne lui appartiendra jamais définitivement.

17. A. Ahmatova, Sobr. soč. v 6 tt., t. 5, p. 121.

I8. Akhmatova a parlé ainsi des Fleurs romantiques de Goumiliev: "Ce livre contient toute l'horreur de cet amour - tous ses cauchemars, son délire et son asphyxie. Le fantôme du suicide suit pas à pas le Poète... A cette époque, Elle devient pour le poète Lilith, c'est-à-dire le principe mauvais et enchanteur de la femme. Il commence à voir en elle une force terrible» (A. Ahmatova, Sobr. soč. v 6 tt., t. 5, p. 115).

I9. «I krotkaja Eva, igruška bogov, / Kogda-to rebenok, kogda-to zarnica, / Teper' dlja nego molodaja tigrica, / V zloveščem mercan’i ee žemčugov, / Predvestnica buri, ikrovi, i strasti, / I radostej zlobnyh, i hmuryh nesčastij.» 


\section{Salomé: Eve et Lilith}

La traduction de la pièce d'Oscar Wilde, réalisée par Constantin Balmont et publiée en 1904, marque en Russie le début d'un engouement pour Salomé. Il traduit de la façon la plus éclatante la philosophie du temps: qu'on pense à la beauté démoniaque, à l'amoralisme esthétique, ou encore à la sensualité charnelle.

La philosophie de Nietzsche, notamment, prône l'idée de la possibilité d'un contact intense avec le monde, par le biais de l'«ivresse" dionysiaque ou de la danse extatique, dans laquelle le corps, gouverné par la sensation immédiate des choses, reconstruit avec sa propre plastique l'agitation des forces élémentaires. Le lien dionysiaque avec l'univers rompt l'autarcie du monde intime, dissout l'être individuel dans le grand tout, et transforme l'individu en simple élément constitutif d'une vie universelle libérée des barrières de la subjectivité. L'ivresse dionysiaque s'éprouve dans l'immédiateté, elle ne peut être "communiquée", mais possède une incontestable force d'entraînement, dont Salomé dans l'art, et Isadora Duncan dans sa vie, sont les meilleures illustrations.

Dans «le tourbillon de la danse saloméenne ${ }^{20}$, les frontières entre le passé et le futur, entre le moral et l'amoral, entre l'amour et la mort, s'effacent.

Qui dit Salomé, dit, certes, danse mais aussi la tête coupée de JeanBaptiste. Si la danse de Salomé entraîne son public dans l'espace indivisible de Dionysos, elle se voit aussi couronnée, au moment de l'extase, par une décapitation, œuvre de la même Salomé. L'interprétation de cet acte dépend, là encore, de ce qui retient l'attention: la tête sans corps chez le symboliste Blok; le corps sans tête chez l'avant-gardiste Maïakovski.

La Salomé d'Alexandre Blok ${ }^{21}$, comme certains critiques l'ont fait remarquer, présente une ressemblance frappante avec l'Hérodiade du court mais célèbre poème intitulé «Le Cantique de Saint-Jean » ${ }^{22}$

20. «...le tourbillon de la danse de Salomé» est une expression d'Anna Akhmatova, qu'elle utilise dans le Poème sans héros.

2I. Sur la figure de Salomé dans l'œuvre de Blok, voir: O. Matič, «Pokrovy Salomei : Eros, smert' i istorija».

22. Ce poème appartient à la troisième partie d'Hérodiade, pièce poétique commencée par Mallarmé en 1864 et fut publié pour la première fois en 1913, donc de nombreuses années après la mort de Mallarmé. 
de Stéphane Mallarmé. Dans ce dernier poème, la muse décapitée libère la voix du poète: la tête de Jean-Baptiste, privée de corps, dit de merveilleux vers et devient la voix de la poésie pure. L'Hérodiade de Mallarmé (qui agit "contre la nature», prive le poète de son essence virile et affranchit sa tête de l'histoire de son corps) donne à la poésie la possibilité de se constituer.

Maïakovski, lui, prend le contrepied de Blok. Dans Le Nuage en pantalon, le premier plan est occupé par un personnage dont le corps se tord, se brise, un personnage qui ne veut être ni montagne de fer ni «nuage en pantalon» désincarné; son rôle évoque, transposé à l'époque de la révolution, celui de Jean-Baptiste et, par là même, le destin de ce dernier. Le héros maïakovskien désire, réclame un corps féminin vivant, de la même façon que les chrétiens demandent leur pain quotidien et le Corps du Christ, ou, cette association s'impose, que Salomé exige la tête de Jean:

\section{Marie!}

Le poète des sonnets chante pour Tian

et moi, je suis entièrement de chair,

un être humain complet -

je demande simplement ton corps ${ }^{23}$.

Que le complexe de Salomé soit un nœud focal majeur du poème de Maïakovski ne devient évident qu'à la fin, lorsque, dans le champ des correspondances sémantiques, des recoupements et des reflets, apparaissent la terre, comparée à la tête de Jean-Baptiste, le soleil, comparé à Hérodiade et à sa danse, et le cour sanglant du poète:

Je mets en joie le chemin du cœur par mon sang

les tuniques de la poussière collent avec des fleurs.

Le soleil dansera mille fois

comme Hérodiade

autour de la terre -

tête de Jean-Baptiste.

Et quand ma quantité d'années

sera tressée jusqu'au bout

23. «Marija! / Poèt sonety poet Tiane, / a ja - ves' iz mjasa, / čelovek ves' - / telo tvoe prosto prošu ", V. Majakovskij, Poln. sobr. soč. v 13 tt., t. 1, p. 193. 
la trace qui conduit à la maison de mon père sera jonchée de millions de gouttes de sang ${ }^{24}$.

En cette période fin de siècle, toutes les combinaisons, répartitions, permutations possibles de ces deux éléments, la tête et le corps, l'esprit et la matière, l'intellect et la nature, de même que toutes les interprétations de ces variantes, sont soigneusement passées en revue.

Le problème de l'équilibre entre la matière et l'esprit et le problème de la femme forment, à cette époque, une sorte de couple métonymique.

Dans la Femme cohabitent Eve et Lilith, sous la forme de deux principes opposés (qui s'attirent et se repoussent simultanément), constamment en lutte. Eve représente le corps, Lilith l'esprit. Jusqu'à un certain point, ces principes s'équilibrent. Mais il arrive que Lilith et Eve se fondent si bien que la Femme se transforme en Salomé. La danse extatique de cette dernière indique précisément que la fusion du corps et de l'esprit, de Lilith et d'Eve a atteint le suprême degré d'intensité qui rend possible le triomphe de la vie dans son unité totale et indivisible. Cependant, le risque existe que la belle vous entraîne du côté opposé, là où se produit, non point l'union du corps et de l'esprit, mais leur séparation. Le danger de la femme réside dans son arbitraire "cinématique»: il suffit parfois d'un seul mouvement maladroit pour que l'Eve ordinaire se change en Salomé extatique, puis, tout aussi imprévisiblement, en Lilith vampirique.

La pièce de Leonid Andreïev, Catherine Ivanovna, montre justement que cette oscillation entre le mystique et le pathologique fait tout le danger de la nature féminine. L'héroïne, en effet, Eve douce et pudique, devient démon. Dans un accès de jalousie, son mari est à deux doigts de la tuer, et ce drame déclenche sa métamorphose. Dans la description que donne Andreïev de son personnage, une attention particulière est portée à sa démarche de danseuse et à ses bras levés qui évoquent des ailes. Catherine Ivanovna se transforme non pas en Salomé, mais en un double de celle-ci, qui n'en possède que le corps:

24. Ibid., p. 194. "Krov'ju serdca dorogu raduju, / lipnet cvetami u pyli kitelja. / Tysjaču raz opljašet Irodiadoj / solnce zemlju - / golovu Krestitelja. / I kogda moe količestvo let / vypljašet do konca - / millionami krovinok usteletsja sled / k domu moego otca.» 
Vous n'êtes pas une bacchante. Vous êtes une chose inerte, morte, et vous vous livrez à la débauche... en rêve! ${ }^{25}$.

La danse de l'héroïne d'Andreïev n'est pas une danse où, dans l'extase, l'âme et le corps fusionnent tout en se dévoilant; elle consiste plutôt en simagrées impudiques d'un corps dénudé, mû non par une âme, mais par les forces obscures de la mort:

Catherine Ivanovna pousse des cris bizarres, tourne, agite sauvagement, désespérément les bras, et soudain s'arrête dans une pose de défi impudique. Ses lèvres sont retroussées en un méchant sourire, ses yeux regardent avec dédain et insolence ${ }^{26}$.

Néanmoins, le plat qu'elle tient reste vide. Contrairement à la vraie Salomé qui brûle d'y voir la tête de Jean, Catherine Ivanovna est disposée à y recevoir n'importe quel chef: sa danse ne célèbre pas la plénitude et l'unité de la vie et de l'amour, elle est l'apothéose de la mort et de la concupiscence omnivores. En outre, tout ce qui arrive à Catherine Ivanovna ne peut être énoncé à l'aide de la formule: " $A$ est $B$ ", mais, comme le dit un des personnages, "tantôt c'est ceci, tantôt c'est cela»:

Il y a, en Catherine Ivanovna, trop de - ah, comment l'exprimer? bref, trop de nature féminine, trop de féminité... Va comprendre ce qu'il lui faut! Ainsi, admettons que je me rende, moi, un homme, dans le royaume des Cieux, je formule la chose telle quelle et tous la voient ainsi : voilà quelqu'un qui va au royaume des Cieux! Mais une femme? Le diable sait où elle va! Tantôt elle se lance dans la débauche, tantôt sa débauche prend des airs dévots... Eternelle MarieMadeleine à laquelle la débauche, commencement ou fin, est absolument nécessaire. La débauche est son Golgotha, sa terreur et son rêve, son paradis et son enfer... ${ }^{27}$.

Dans les entretiens de Nikolaï Molostvov et d'Akim Volynski sur Isadora Duncan, est exprimée la crainte que, la technique de la danseuse étant imparfaite, ses défauts ne finissent par devenir son «talon

25. L. Andreev, Dramatičeskie proizvedenija v 2 tt., t. 2, p. 112.

26. Ibid., p. 127.

27. Ibid., p. 118. 
d'Achille» et que "l'idée fondant sa danse ne vole en éclats, laissant le simple souvenir d'une "grande attraction" exotique à la mode» ${ }^{28}$. Soulignons que l'exotique est ici opposé, non au national, à ce qui appartient en propre, mais à l'idéel, à l'intime, au plus profond. Il est très significatif que la danse de Duncan, regorgeant d'expressions et de couleurs orientales, à l'instar de la danse de Salomé, est, pour la sensibilité fin de siècle, non la manifestation la plus élevée du concept d'exotisme (tel qu'on le trouve, par exemple, dans L'Orient de Théophile Gautier ou dans Le Voyage en Orient de Gérard de Nerval), mais son exact contraire. La sémiotique est ici tout autre: Isadora Duncan, nu-pieds, à demi-dévêtue; Ida Rubinstein, qui évoque une momie égyptienne dans le ballet de Fokine La Nuit de Cléopâtre; Natalia Volokhova, la vierge assassine dans la mise en scène de Salomé par Nikolaï Evreïnov; l'inconnue de Blok, visage moderne sur lequel se surimpose celui de la femme antique; les fantastiques décors et costumes de Léon Bakst - autant de manifestations ouvertement exotiques, voire d'un exotisme provocant, qui s'efforcent en même temps d'exprimer ce qui n'est pas exotique.

Pour parler de leur universalité (qui exclut toute distinction temporelle, spatiale, historique ou ethnographique), la littérature et la culture du début du XXe siècle ont recours au langage imagé de l'exotique. C'est là qu'apparaît la profonde signification de l'exotisme comme "négation de tout caractère historique concret " ${ }^{29}$. L'imagerie exotique fin de siècle ne dessine plus de frontières séparant le familier de l'étranger, elle montre l'univers et la femme, son double métaphorique aussi bien que métonymique. L'univers ne peut être considéré ni comme familier ni comme étranger, car il est à la fois l'un et l'autre. Et la question est de savoir appréhender ce qui ressortit au familier, avant qu'il ne se change en étranger, et de distinguer les deux.

Dans un décor exotique, Salomé, en se dépouillant de ses sept voiles au rythme de sa danse extatique, se libère symboliquement des couches de culture et d'histoire, et se désigne elle-même, dans sa nudité et sa nature féminine ambivalente, comme l'unique, l'authentique et l'éternel sujet exotique, comme l'unique, l'authentique et l'éternel objet d'expérience exotique.

28. N. G. Molostvov, Ajsedora Dunkan, p. 6.

29. C'est ainsi que Roland Barthes a défini la profonde signification de l'exotisme dans l'un de ses essais dans Mythologies. Cf. R. Barthes, Mifologii, p. 236-237. 
De nouveau, comme dirait Anna Akhmatova, "ressurgit» devant Adam le " "problème de la femme" (Eve et Lilith, la sainte et la pécheresse). Et l'effroi : "une étrangère, une étrangère!" "30.

\author{
Sergueï Savinkov \\ Voronèje \\ Traduction Corinne Fournier Kiss
}

30. A. Ahmatova, Sobr. soč. v 6 tt., t. 5, p. 121. 


\section{BIBLIOGRAPHIE}

Aнmatova, A., Sobr. soč. v 6 tt., Moskva, 2001.

Andreev, L., Dramatičeskie proizvedenija v 2 tt., Leningrad, 1989.

Barthes, R., Mifologii, Moskva, 2008.

Beljaev, A., Sobr. soč. v9 tt., Moskva, 1993.

Cvetaeva, M., Sob. soč v 7 tt., Moskva, 1994.

Gumilev, N., Sočinenija v 3 tt., Moskva, 1991.

Majakovskij, V., Poln. sobr. soč. v 13 tt., Moskva, 1955.

Matič, O., "Pokrovy Salomei: Eros, smert' i istorija", in Erotizm bez beregov, Moskva, 2004.

Misnikevič, T. V., "Fedor Sologub, ego poklonnicy i korrespondentki ", in Erotizm bez beregov, Moskva, 2004.

Molostvov, N. G., Ajsedora Dunkan (beseda s A. A. Volynskim), SanktPeterburg, 1908.

Naвokov, V., Poems and Problems, New York, McGraw Hill, 1970.

—, Stihotvorenija i poèmy, Moskva, 1997.

Nietzsche, F., Volja k vlasti, Moskva, 1994.

Severjanin, I., Stihotvorenija i poèmy, Moskva, 1990.

Sologub, F., Sobr. soč., Moskva, 2004.

VRANGEL', N. N., «Ljubovnaja mečta sovremennyh russkih hudožnikov», Apollon, 3 (1909), p. 32-45. 
